\title{
Medical Image Processing Methodology for LiVER TuMOUR Diagnosis
}

\author{
Thayalini Prakash \\ Department of Software Engineering, University of Westminster, UK
}

\begin{abstract}
Apply the Image processing techniques to analyse the medical images may assist medical professionals as well as patients, especially in this research apply the algorithms to diagnose the liver tumours from the abdominal CT image. This research proposes a software solution to illustrate the automated liver segmentation and tumour detection using artificial intelligent techniques. Evaluate the results of the liver segmentation and tumour detection, in-cooperation with the radiologists by using the prototype of the proposed system. This research overcomes the challenges in medical image processing. The 100 samples collected from ten patients and received $90 \%$ accuracy rate.
\end{abstract}

\section{KEYWORDS}

CT abdominal image, Medical Image processing, liver segmentation, Tumour region extraction

\section{INTRODUCTION}

In this ear, Artificial intelligence, image processing and machine learning techniques are heavily used in the medical world to analyse the medical images and diagnose the tumour, such as Ultrasound, MRI and CT scans images can be analysed by the computer aided tool. As stated by Radiological Society of North America, Inc., 2010; the CT imaging is one of the finest and fastest techniques to identify the abdominal and pelvis due to high-quality, also screening the crosssectional view of entire tissue. On top of this, fully-automated system for remedial diagnoses is getting demand among medical experts. This research engages to overcome the existing problem and propose a fully automated system for liver segmentation and tumour detection from an abdominal CT image.

\section{METHODOLOGY}

\subsection{LIVER SEgMENTATION}

Identifying liver from CT abdominal image is generally a challenging task due to other organs with same intensity as liver, adjacent to the liver makes segmentation more difficult. Nonetheless, as define by Zayane. O, et al., (2011), and according to the anatomic studies (Abrahams, P., 2002), the liver is constantly locates at the upper right side of the abdomen and takes up the largest area among the various organs included in the abdomen image. In this experiment liver region will be extracted by using the following 2 steps.

In the first step: Using anatomical knowledge of the liver and histogram analyser, adaptive threshold is fixed to extract the liver pixels from the CT abdominal image which usually contains other organs including stomach, kidney, spleen, pancreas, etc. 
In the second step: Morphological operations like closing and opening operations are used to preserve the structure of the liver and to remove the small fragments of other organs adjacent to the liver with the same intensity as that of liver.

This research recommends the following approaches to segment the liver and tumour region with more affluence and accurate manner. The original CT abdomen image has been processed through the steps as shown in the below illustration.

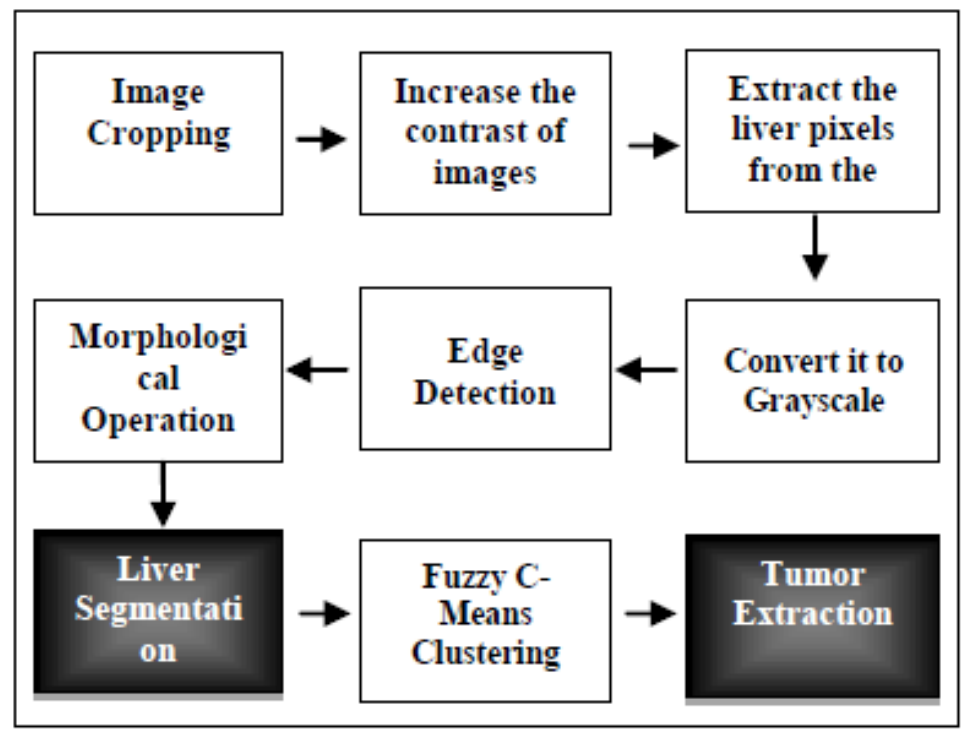

Figure 1: Automatic Liver and Tumour Region Extraction Approach

\subsubsection{Image Cropping}

Each image was cropped before any processing or visualization was performed. The main reason for doing this was to decrease the computational time, but there was also the added benefit in that focuses on only the target area liver and it became the main organ in each image. Each image was cropped at rows 30 and 190 and columns 15 and 240. Referring to figure 2, it can be seen how cropping the image resulted in placing the liver as the focus of the CT scan. For the CT abdominal image, Resize the input image and a window is fixed by using cropping technology to remove the last 30 rows and 15 columns from the right since this area usually does not contain liver region.

The liver is extracted along with the fragments of other organs located near to it and with the intensity same as that of liver. Based on certain conditions, the fragments of adjacent organs can be removed.

The first condition is the location of the object: The liver is placed at a constant area in the upper right side of the image.

The second condition is the area: The area of the liver is large when it is compared with the fragments of other organs. After removing the fragments, the image obtained is complemented and multiplied with the original image to get the segmented liver in the CT abdominal image. 


\subsubsection{Histogram Equalization}

Histogram Equalization works by improving the contrast of images by stretching the intensity distribution of the image or by changing the values in a colour map being used. An image can then be produced that approximately matches the specified histogram. The image that received by running a Histogram Equalization on this image can be seen in Figure 2. The change is quite noticeable as this slice is the same slice. It can be seen that the image detail is more apparent and that the tumour and blood vessels can be seen much more easily than before. It is experimented with 100 images and supported with $90 \%$ accurate.

\subsubsection{Adaptive threshold}

The liver region maintains a constant intensity throughout, a fixed threshold is impossible because the intensity differ from patient to patient also depends on the quality of a CT image. Therefore, a system has to be developed to extract the liver automatically with the adaptive threshold decision.

The threshold is called adaptive threshold when a different threshold is used for different regions in the image, as stated above, can't use the same threshold for the entire image, thus this research recommends that the adaptive threshold is appropriated.

\subsubsection{Convert it to Grayscale}

Converts the true colour image RGB to the grayscale by eradicating the hue and saturation information while retentive the luminance. RGB values to grayscale values by calculating a prejudiced sum of the $\mathrm{R}, \mathrm{G}$, and $\mathrm{B}$ components:

$0.2989 * \mathrm{R}+0.5870 * \mathrm{G}+0.1140 * \mathrm{~B}$

\subsubsection{Edge detection}

Yu-qian.Z., et al. (2005) recommended a novel mathematic morphological algorithm for lung detection from CT image. They acknowledged that, this is as a better algorithm for medical image detection and de-noising than the template-based edge detection algorithms and general morphological edge detection algorithm. Nevertheless, Hiransakolwong et al., (2003), and Maini.R and Sobel.J.S., (2006), proposed the Sobel edge detection for medical image segmentation. However, according to the author's experiments of edge detection algorithms for 100 samples, "Sobel" supports $80 \%$ while "Canny" supports $40 \%$. As a result, this research recommends the "Sobel" algorithm for liver segmentation.

\subsubsection{Morphological Operation}

Morphological operation has been applied in the research as part of the liver segmentation process; the octagonal structuring element approach segmented with only $30 \%$ of accuracy, for another $70 \%$ of images segmented both liver and kidney. However, disk-shaped structuring element approach supports with 70\% accuracy. This research recommends the disk function based on the experimental result.

Erode image - Erode the filled image so as to leave just the liver. Each image was eroded using a disk of size 20 to erode with. Create morphological structuring element.

Erodes the grayscale, binary, or packed binary image, returning the eroded image. The argument se is a structuring element object or an array of structuring element objects returned. 
International Journal on Soft Computing (IJSC) Vol.8, No. 3/4, November 2017

If a binary image is logical and the structuring element is flat, performs binary erosion; otherwise it performs grayscale erosion. If structuring element is an array of structuring element objects, performs multiple erosions of the input image, using each structuring element in succession.

Multiplied with original CT abdominal Image, Modified regional maxima super imposed on the original image.

\subsection{TUMOUR EXTRACTION}

Automatic tumour extraction also a challenging task, due to variety of difficulties such as tumour shape variations, low contrast between tumour tissue and normal liver tissue, different tumour imaging characteristics at different CT scanning phases.

Hong. J.S, et al. (2001) proposed a CAD system based on Fuzzy C Means Clustering for liver tumour extraction with an accuracy of $91 \%$ using features like area, circularity and minimum distance from the liver boundary to tumour and Bayes classifier for classifying normal and abnormal slice. Also, Lee.C.C, et al. (2003) identified liver region by using the fuzzy descriptors and fuzzy rules constructed using the features like location, distance, intensity, area, compactness and elongated-ness from CT abdominal images.

From the liver, tumour region is segmented using, FCM clustering technique. FCM minimizes the object function through the iterative optimization of the membership function based on the similarity between the data and the centre of a cluster. FCM varies the threshold between clusters through an iterative process. As a result, the threshold is determined appropriate for every slice and the tumour region can be successfully extracted.

The pixels of the input image are divided into three clusters. The first cluster includes pixels in the background (low intensity). The second cluster includes pixels in the tumour region (medium intensity) and the third cluster includes pixels in the liver region other than tumour (high intensity). The tumour region is outputted for further analysis.

\subsubsection{Otsu's method}

The graythresh function uses the Otsu's method, which chooses the threshold to minimize the intraclass variance of the black and white pixels.

\subsubsection{Outline the Liver in original Image}

The Liver region would be highlighted through draw a skeleton on the segmented liver potion. The outline is shaped by the bwperim function. Imoverlay function also does the same process but it won't support to 3D images that's why unable to apply this function. Also, Segout is reassigned as the cropped image and then the outline places on the cropped image.

\subsubsection{Texture Feature Extraction}

Texture feature is a commonly use characteristics in medical image interpretation, also applicable to a variety of image processing problems. However, John.E.K et al, (1999) stated that, the tissues are expected to have consistent and homogeneous textures along with the series of slices. Therefore, texture information can be practiced to categorize among different organ's tissues. Also, gray level co-occurrence matrices estimate the properties of images related to second-order statistics. The Gray level co-occurrence matrices are known as a widely used approach for texture analysis, Kurani. A.S. et al. (2004) recommended the GLCM techniques for better feature extraction. 


\section{EXPERIMENT}

The approach has been experimented with CT abdomen image and the expected result was archived. The experiment results as shown below:

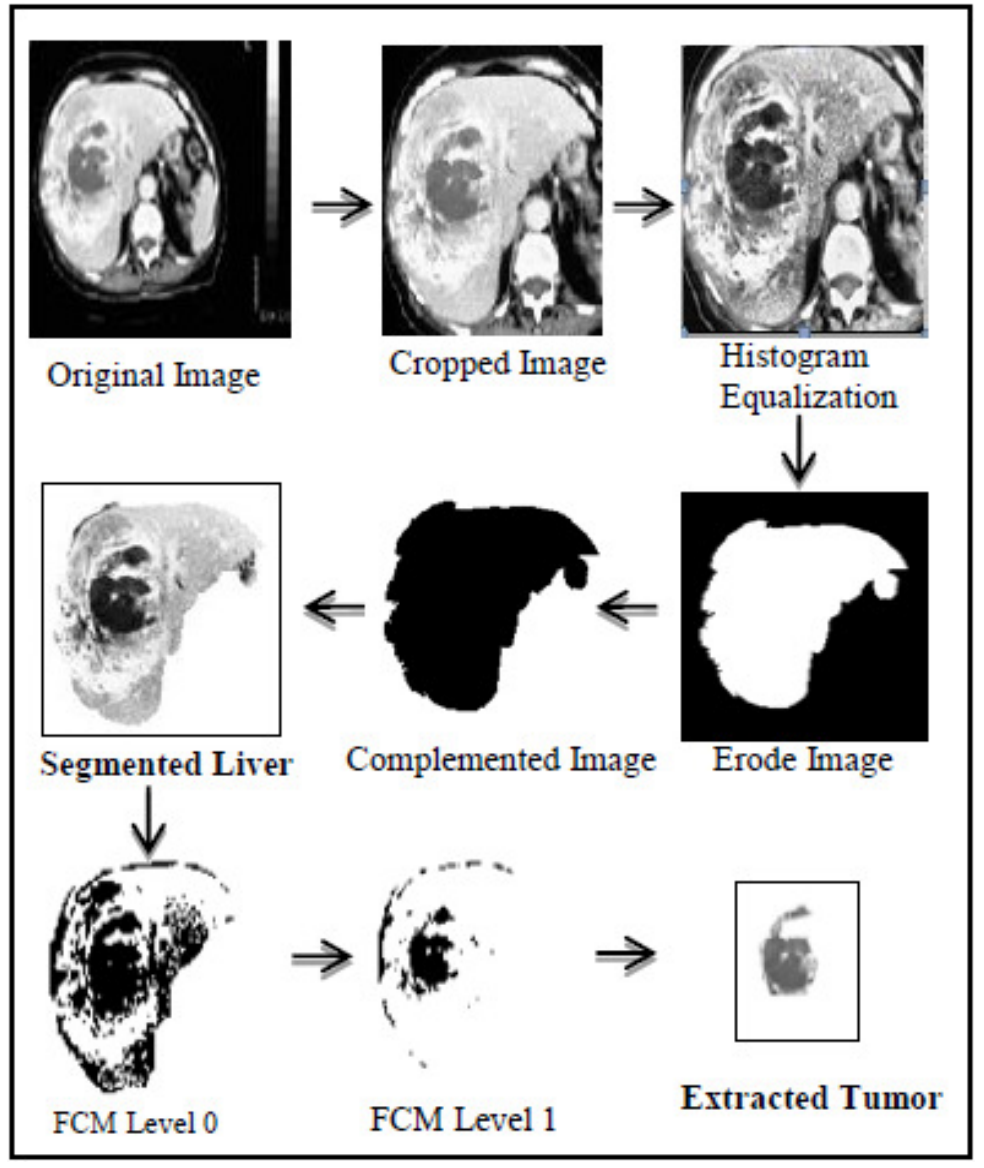

\section{EvAluation}

Figure 2: Experiment Result

Each sub modules are experimented separately with 100 samples and the experiment result received as follows: the pre-processing stage, Change the size, contrast of the Image given $96 \%$ accuracy and Main experiment of the research; liver segmentation from the inputted CT abdomen image successfully resulted with $90 \%$ accuracy, likewise, another major task, extract the tumour region from segmented liver image achieved with 92\% accuracy, Finally Extract the value of mean, standard, homogeneity, entropy, energy, contrast, correlation also resulted as expected with $94 \%$ accuracy level.

The performance and accuracy of end product evaluated with the support of radiologists, such as accuracy, predictability, usability, functionality, user interface and user friendliness. Additionally, the concept and technology of the product are evaluated by the computer technical experts. Finally, the most important factor of a medical product, 'accurately' assessed with $100 \mathrm{CT}$ abdomen images gathered from ten different patients, and received $90 \%$ of accuracy. The bellows formula practiced to figure out the accuracy level. 


$$
\text { Accuracy }=\frac{\text { Total No of Correctly Segmented Image }}{\text { Total No of Tested Image }} * 100
$$

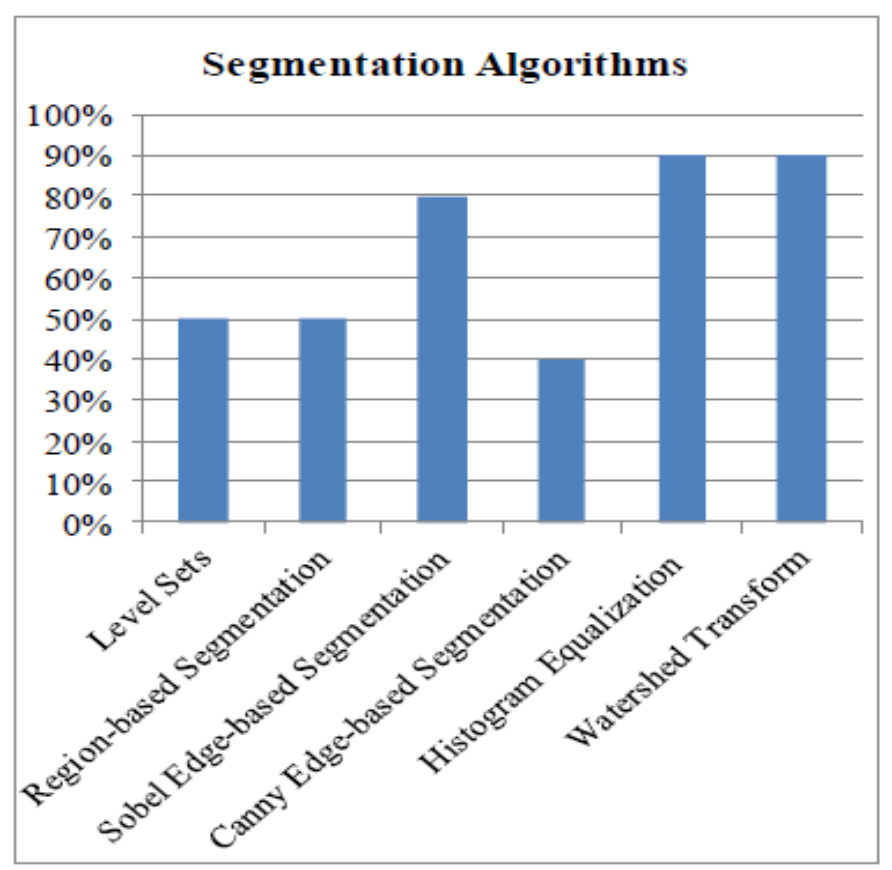

Figure 3: Evaluation Result

According to the experiment and evaluation result, out of these above technologies Sobel Edge detection, Histogram Equalization, Watershed Transform methods are recommended by the author as part of the process of automatic liver segmentation from CT abdomen images. Further, Region-based segmentation appropriate for semi-automatic approach and Level set algorithm works as semi-automatic; it will detect the liver when the user manually clicks on the liver area. However it's segmented the liver with some other organs.

\section{CONCLUSION}

In this research, the liver region automatically segments from CT abdominal images, using the anatomical knowledge of the liver, adaptive threshold decision based on intensity information and morphological processing in watershed method. Tumour region extracts by using Fuzzy C Means Clustering (FCM). Gray Level Co-occurrence Matrix practice for extract the texture features. All these technologies and algorithms are well examined and critically evaluated.

\section{RECOMMENDATION}

Recommend to expand this research up to classify the tumour category and compute the size of the tumour in the future. It might help to reduce the radiologist's work load as well it will be a better guidance for patient to track and understand their tumor cure probability. 


\section{ACKNOWLedGement}

Author wish to thank radiologist who shares the domain knowledge and samples of CT abdomen images, in addition to that would like to thank medical experts and technical experts who evaluate the approach. Without their involvement, it would not have been successes.

\section{REFERENCES}

[1] Abrahams, P., 2002. The Atlas of the Human Body. 2nd ed. San diego, California, USA: Bright Star Publishing Plc.

[2] Hiransakolwong.N., Windyga.P.S., Hua.K.A., and Khanh.V., 2003. Segmentation of ultrasound liver images: an automatic approach. [Online] Available at: http://www.computer.org/portal/web/csdl/doi/10.1109/ICME. 2003.1220982 [Accessed 10 Dec 2012].

[3] Hong.J.S., Kaneko.T., Sekiguchi.R., and Houmpark.K.,(2001). Automatic Liver Tumor Detection from CT. IEICE Trans. Inf.\& Syst, E84-D(6), pp. 741-748.

[4] John.E.K., Newman. F.D., Johnson.T.K., and Kirch. D.L., 1999. Abdominal Organ Segmentation Using Texture Transforms and a Hopfield Neural Network. IEEE Transactions on Medical Imaging, 18(7), pp. 640-648.

[5] Kurani AS, Xu DH, Furst J, Raicu DS., 2004. Co-occurrence Matrices for Volumetric Data. Hawaii, USA, s.n.

[6] Lee.C.C, Chung.P.C., and Tsai.H.M., 2003. Identifying Abdominal organs from CT image series using a Multimodule Contextual Neural network and Spatial Fuzzy rules. IEEE Transactions on Information Technology in Biomedicine, 7(3), pp. 208-217.

[7] Maini.R and Sobel.J.S., 2006. Performance Evaluation of Prewitt Edge Detector for Noisy Images. GVIP Journal, 6(3).

[8] Mala.K., Sadasivam.V., and Alagappan.S., "Neural Network based Texture Analysis of Liver Tumour from Computed Tomography Images," World Academy of Science, Engineering and Technology International Journal of Medical, Health, Biomedical, Bioengineering and Pharmaceutical Engineering Vol:2, No:1, 2008.

[9] Radiological Society of North America, Inc., 2013. RadiologyInfo.org. [Online] Available at: http://www.radiologyinfo.org/en/about/index.cfm?pg=abt_co pyrt [Accessed 16 Mar 2013].

[10] Yu-qian.Z., Wei-hua.G., Zhen-cheng.C., Jing-tian.T., Ling-yun.L., 2005. Medical Images Edge Detection Based on Mathematical Morphology. IEEE - Engineering in Medicine and Biology Society, p. $6492-6495$.

[11] Zayane.O., Jouini1.B., and Mahjoub.M.A., 2011. Automatic liver segmentation method in CT images. Canadian Journal on Image Processing \& Computer Vision, 2(8). 